\title{
Reducing the impact of measurement errors in FRF-based substructure decoupling using a modal model
}

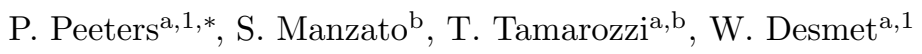 \\ ${ }^{a}$ KU Leuven, Department of Mechanical Engineering Celestijnenlaan 300, B-3001, \\ Heverlee, Belgium \\ ${ }^{b}$ Siemens Industry Software NV, Interleuvenlaan 68, B-3001, Leuven, Belgium
}

\begin{abstract}
As the vibro-acoustic requirements of modern products become more stringent, the need for robust identification methods increases proportionally. Sometimes the identification of a component is greatly complicated by the presence of a supporting structure that cannot be removed during testing. This is where substructure decoupling finds its main applications. However, despite some recent advances in substructure decoupling, the number of successful applications has so far been limited. The main reason for this is the poor conditioning of the problem that tends to amplify noise and other measurement errors.

This paper proposes a new approach that uses a modal model to filter the experimental frequency response functions (FRFs). This can reduce the impact of noise and mass loading considerably for decoupling applications and decrease the quality requirements for experimental data. Furthermore, based on the uncertainty of the observed eigenfrequencies, an arbitrary number of consistent (all FRFs exhibit exactly the same poles) FRF matrices can be generated that are all contained within the variation of the original measurement. This way, the variation that is observed within the measurement is taken into account. The result is a distribution of decoupled FRFs of which the average can be used as
\end{abstract}

\footnotetext{
* Corresponding author

Email address: pepijn.peeters@kuleuven.com (P. Peeters)

${ }^{1}$ Member of Flanders Make
}

Preprint submitted to Journal of Mechanical Systems and Signal Processing July 27, 2017 
the decoupled FRF set while the spread on the results highlights the sensitivity or reliability of the obtained results.

After briefly reintroducing the theory of FRF-based substructure decoupling, the main problems in decoupling are summarized. Afterwards, the new methodology is presented and tested on both numerical and experimental cases.

Keywords: Substructure decoupling, Experimental dynamic substructuring, Frequency based substructuring, Noise filtering

\section{Introduction}

Since the end of the 60's, dynamic substructuring techniques are being developed to analyse large structures and systems by dividing them into smaller, more manageable parts and coupling them in order to obtain the static and dynamic behaviour of the total system [1]. Although substructuring techniques are generally used to couple components, sometimes substructure decoupling, or the addition of a fictitious "negative structure" [2], can be useful. For example, when a component is too heavy or fragile to suspend freely or when it is integrated in a drive-train, its dynamics are influenced by the supporting structure. Substructure decoupling techniques offer an experimental approach to assess the dynamic behaviour of an individual component that is part of a larger system.

Earlier publications by Ind et al. in 2003 [3] and D'Ambrogio and Fregolent in 2004 [4] focused on a modal approach for substructure decoupling. Also Allen, Mayes and Kammer used a modal approach to couple and uncouple components with the so-called transmission simulator method $[5,6,7]$. In 2005, D'Ambrogio [8] introduced an FRF-based approach similar to the structural modification procedure [9]. In 2008, Sjövall and Abrahamson presented a decoupling method based on reconstruction of the interface forces acting between the substructures. They found that the method is most sensitive to the existence of general anti-resonances in the frequency domain of interest [10]. In the next years, a more general framework for substructure decoupling was presented 
by D'Ambrogio and Voormeeren describing the primal and dual approach for substructure decoupling and a family of decoupling techniques, this time solely relying on experimental data $[2,11,12,13]$. Research that followed showed that (rotational) connection degrees of freedom (DoFs) do not need to be measured explicitly if additional DoFs internal to the test rig are measured [14, 15, 16, 17].

However, despite these advances, the number of successful decoupling applications remains limited. This is mainly due to the poor conditioning of the problem that tends to amplify noise and other errors in the measurements $[2,13,14,15]$. Some authors have attempted to filter the FRFs before inversion to reduce the detrimental effects of noise. In 2004 Sanliturk and Cakar [18] used a so-called Hänkel matrix in combination with an truncated singular value decomposition (TSVD) to filter FRFs. The filtering results are fairly successful, but the method is hard to automate and can become expensive due to the TSVD [18]. In [14] D'Ambrogio and Fregolent used the poly-reference least-square complex frequency domain (pLSCF) method [19, 20], to curve fit a modal model on noisy FRFs. A significant reduction in accuracy was reported in the decoupled FRFs. Moreover, in [16] the pLSCF technique was used to filter the experimentally obtained FRF matrices in a global way. Although the reconstruction of the original FRFs was fairly good, the decoupling results were reportedly not satisfactory. In a previous publication [21] a common denominator polynomial model is used to filter the FRFs and impose consistency at the same time. The principle is similar to the approach presented in this paper. The filtering capabilities of the polynomial model are good for different types of noise, but mass loaded FRFs remain problematic.

In this paper, a new method is proposed that uses a modal model to filter the FRFs in a column-wise approach while enforcing consistency throughout the FRF matrix of assembly and residual system. Consistency is achieved by imposing identical eigenfrequencies for similar poles throughout the FRF matrix. This way the impact of systematic errors, like e.g. mass loading or a change in boundary conditions, is greatly reduced. Furthermore, based on the observed variation of the eigenfrequencies in the experimental data, an arbitrary number 
of consistent FRF matrices can be generated that are all contained within the variability of the original measurements. This way, the variation that is observed within the measurement is taken into account. The result is a distribution of decoupled FRFs of which the average can be used as the decoupled FRF set while the spread on the results highlights the reliability of the obtained results.

This paper will first briefly reintroduce the dual approach for substructure decoupling. Then some of the main difficulties encountered in the method are discussed. After that, the new filtering approach is presented. First the main characteristics of the new approach are described and then the different calculation steps are explained more in detail. The method is then tested on both simulated and experimental results.

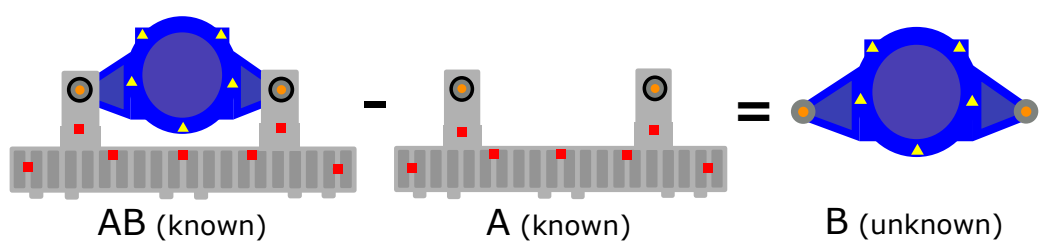

Figure 1: Principle of substructure decoupling

\section{Substructure decoupling}

This section will first briefly discuss the dual approach for substructure decoupling. Afterwards, some of the experimental difficulties encountered with substructure decoupling are discussed.

The principle of substructure decoupling is shown in figure 1. The DoFs of the assembled system can be divided into 3 groups: DoFs internal to the supporting structure A, DoFs internal to the decoupled component B and DoFs connecting the (two) substructures. The DoFs are illustrated as squares, triangles and dots respectively in figure 1. 


\subsection{Dual formulation for substructure decoupling}

The decoupling problem can be described using the dynamic stiffness representation of the subsystems $[12,13]$. The assembly can be expressed as:

$$
\begin{gathered}
\boldsymbol{Z}^{A B} \boldsymbol{u}^{A B}=\boldsymbol{f}^{A B}-\boldsymbol{g}^{A B} \\
{\left[\begin{array}{lll}
\boldsymbol{Z}_{a a}^{A B} & \boldsymbol{Z}_{a c}^{A B} & \boldsymbol{Z}_{a b}^{A B} \\
\boldsymbol{Z}_{c a}^{A B} & \boldsymbol{Z}_{c c}^{A B} & \boldsymbol{Z}_{c b}^{A B} \\
\boldsymbol{Z}_{b a}^{A B} & \boldsymbol{Z}_{b c}^{A B} & \boldsymbol{Z}_{b b}^{A B}
\end{array}\right]\left[\begin{array}{l}
\boldsymbol{u}_{a}^{A B} \\
\boldsymbol{u}_{c}^{A B} \\
\boldsymbol{u}_{b}^{A B}
\end{array}\right]=\left[\begin{array}{c}
\boldsymbol{f}_{a}^{A B} \\
\boldsymbol{f}_{c}^{A B} \\
\boldsymbol{f}_{b}^{A B}
\end{array}\right]-\left[\begin{array}{c}
\mathbf{0} \\
\boldsymbol{g}_{c}^{A B} \\
\mathbf{0}
\end{array}\right]}
\end{gathered}
$$

In this equation, $\boldsymbol{u}^{A B}$ is a displacement vector, $\boldsymbol{Z}^{A B}$ the dynamic stiffness matrix and $\boldsymbol{f}^{A B}$ the external force vector. The vector $\boldsymbol{g}^{A B}$, the only unknown in equation 1 , represents a disconnection force applied at the connections. The subscripts a, b and c denote the test rig, component and connection DoFs respectively. To determine the interface forces, an identical, but opposite force $\boldsymbol{g}^{A}$ is applied to the empty test rig to make it behave as if it were loaded by the component $[12,13]$ :

$$
\begin{gathered}
\boldsymbol{Z}^{A} \boldsymbol{u}^{A}=\boldsymbol{f}^{A}+\boldsymbol{g}^{A} \\
{\left[\begin{array}{cc}
\boldsymbol{Z}_{a a}^{A} & \boldsymbol{Z}_{a c}^{A} \\
\boldsymbol{Z}_{c a}^{A} & \boldsymbol{Z}_{c c}^{A}
\end{array}\right]\left[\begin{array}{l}
\boldsymbol{u}_{a}^{A} \\
\boldsymbol{u}_{c}^{A}
\end{array}\right]=\left[\begin{array}{l}
\boldsymbol{f}_{a}^{A} \\
\boldsymbol{f}_{c}^{A}
\end{array}\right]+\left[\begin{array}{c}
\mathbf{0} \\
\boldsymbol{g}_{c}^{A}
\end{array}\right]}
\end{gathered}
$$

Similar to equation $1, \boldsymbol{u}^{A}$ is the displacement vector of the empty test rig, $\boldsymbol{Z}^{A}$ the dynamic stiffness matrix and $\boldsymbol{f}^{A}$ an external force vector. The connection DoFs of the assembly are decoupled when both the compatibility and equilibrium condition are satisfied at the interfaces. With the use of a Boolean matrix, the compatibility and equilibrium condition can be expressed as [12, 13]:

$$
\begin{gathered}
\boldsymbol{B} \boldsymbol{u}=\left[\begin{array}{ll}
\boldsymbol{B}^{A B} & \boldsymbol{B}^{A}
\end{array}\right]\left[\begin{array}{c}
\boldsymbol{u}^{A B} \\
\boldsymbol{u}^{A}
\end{array}\right]=\boldsymbol{u}_{c}^{A B}-\boldsymbol{u}_{c}^{A}=\mathbf{0} . \\
\boldsymbol{L}^{T} \boldsymbol{g}=\left[\begin{array}{ll}
\boldsymbol{L}^{A B^{T}} & \boldsymbol{L}^{A^{T}}
\end{array}\right]\left[\begin{array}{c}
\boldsymbol{g}^{A B} \\
\boldsymbol{g}^{A}
\end{array}\right]=\boldsymbol{g}_{c}^{A B}+\boldsymbol{g}_{c}^{A}=\mathbf{0}
\end{gathered}
$$


In the dual approach for substructure decoupling, the equilibrium condition is satisfied a priori by choosing the interface forces in the form $\boldsymbol{g}=\left[\begin{array}{ll}\boldsymbol{g}^{A B} & \boldsymbol{g}^{A}\end{array}\right]^{T}=$ $\boldsymbol{B}^{T} \boldsymbol{\lambda}$.

$\boldsymbol{\lambda}$ are Lagrange multipliers that correspond physically to the interface force intensities $[2,12,13]$. After reordering, the following expression is obtained [2]:

$$
\left[\begin{array}{ccc}
\boldsymbol{Z}^{A B} & \mathbf{0} & \boldsymbol{B}^{A B^{T}} \\
\mathbf{0} & \boldsymbol{Z}^{A} & -\boldsymbol{B}^{A^{T}} \\
\boldsymbol{B}^{A B} & \boldsymbol{B}^{A} & \mathbf{0}
\end{array}\right]\left[\begin{array}{c}
\boldsymbol{u}^{A B} \\
\boldsymbol{u}^{A} \\
\boldsymbol{\lambda}
\end{array}\right]=\left[\begin{array}{c}
\boldsymbol{f}^{A B} \\
\mathbf{0} \\
\mathbf{0}
\end{array}\right]
$$

The explicit distinction can be made between the Boolean matrices enforcing compatibility and those enforcing equilibrium, resulting in expression 5, where $\boldsymbol{E}^{*}$ is the Boolean for equilibrium and $\boldsymbol{C}^{*}$ the Boolean for compatibility [12].

$$
\left[\begin{array}{ccc}
\boldsymbol{Z}^{A B} & \mathbf{0} & \boldsymbol{E}^{A B^{T}} \\
\mathbf{0} & \boldsymbol{Z}^{A} & -\boldsymbol{E}^{A^{T}} \\
\boldsymbol{C}^{A B} & \boldsymbol{C}^{A} & \mathbf{0}
\end{array}\right]\left[\begin{array}{c}
\boldsymbol{u}^{A B} \\
\boldsymbol{u}^{A} \\
\boldsymbol{\lambda}
\end{array}\right]=\left[\begin{array}{c}
\boldsymbol{f}^{A B} \\
\mathbf{0} \\
\mathbf{0}
\end{array}\right]
$$

When the Lagrange Multipliers $\boldsymbol{\lambda}$ are eliminated and the inverse of the dynamic stiffness is replaced by the receptance, i.e.: $\boldsymbol{Z}^{*-1}=\boldsymbol{H}^{*}$, the decoupled response vector $\boldsymbol{u}^{A B}$ can be written as [12]:

$\boldsymbol{u}_{d e c}^{A B}=\left(\boldsymbol{H}^{A B}-\boldsymbol{H}^{A B} \boldsymbol{E}^{A B^{T}}\left(\boldsymbol{C}^{A B} \boldsymbol{H}^{A B} \boldsymbol{E}^{A B^{T}}-\boldsymbol{C}^{A} \boldsymbol{H}^{A} \boldsymbol{E}^{A^{T}}\right)^{+} \boldsymbol{C}^{A B} \boldsymbol{H}^{A B}\right) \boldsymbol{f}^{A B}$

In this vector, the DoFs of substructure B show the decoupled response. Note that ${ }^{+}$denotes the (Moore-Penrose) pseudo-inverse, since the term is not necessarily square or of full rank [12]. Equivalently, the transfer function matrix can be written from which the sub-matrix representing substructure B is the decoupled transfer function [12]:

$$
\boldsymbol{H}_{d e c}=\boldsymbol{H}^{A B}-\boldsymbol{H}^{A B} \boldsymbol{E}^{A B^{T}}\left(\boldsymbol{C}^{A B} \boldsymbol{H}^{A B} \boldsymbol{E}^{A B^{T}}-\boldsymbol{C}^{A} \boldsymbol{H}^{A} \boldsymbol{E}^{A^{T}}\right)^{+} \boldsymbol{C}^{A B} \boldsymbol{H}^{A B}
$$

In fact, as shown in inter alia $[2,13,17]$, the compatibility and equilibrium conditions can also be enforced on locations internal to the test rig, and thus not only at the connections. This method is called the extended interface method 
[13]. This opens possibilities as the DoFs on the test rig can replace some of the (rotational) connection DoFs that are usually hard to measure. This approach is called the mixed interface approach [2]. This can be intuitively understood because the difference between the loaded and unloaded test rig is considered. A force at the connections will also influence DoFs internal to the (flexible) test rig. Therefore the difference in response at other locations than the connections can be considered to carry equivalent information as the responses captured at the interfaces. Furthermore it has been shown that compatibility and equilibrium don't have to be enforced on the same locations $[2,17]$. In practice this means that the excitation and response locations can be non-collocated, eliminating the explicit need for driving-point FRFs.

\subsection{Problems in substructure decoupling}

From a theoretical point of view, the FRF-based decoupling approach is interesting as there is a certain freedom in the choice of measurement locations. Furthermore, no simulations are required. This makes it potentially a very powerful method for the identification of prototypes and other components of which no reliable models are immediately available. However, many other difficulties,

of which some are shared with substructure coupling [1,22], still remain. The most important issues for decoupling are discussed below:

\section{Measurement noise:}

Noise on the measurements is to some extent unavoidable. Especially FRFs between lowly coupled points and directions are the most susceptible to noise as only a little amount of the input energy is captured by the output sensor. Moreover, when a hammer is used, the frequency content of the excitation signal may be hard to control.

2. Systematic errors (e.g. mass loading and frequency shifts):

The use of a shaker allows a better control over the excited frequencies. However, most often the shaker(s) will be moved around the structure in order to measure sufficient columns of the FRF matrix. This can make the measurements to some extent inconsistent with each other as the shaker(s) 
may cause significant mass loading, especially to light structures. This causes frequency shifts between the different columns of the FRF matrix [23]. As a result, spurious peaks may be present in the decoupled FRFs $[24]$.

3. Non-linearities:

Non-linearities may cause the subtraction of assembly and empty test rig to be dissimilar to the free component as depicted in figure 1. This can be caused for example by non-linearities in joints or preloading in bolted connections. Also this can cause spurious peaks in the decoupled FRFs $[24]$.

4. The conditioning number and the selection of measurement locations:

Despite the use of over-determination, the condition number generally remains very high. Moreover, the selection of measurement locations influences the condition of the inversion and therefore the amplification of errors $[11,12,15]$. A good selection of measurement locations is therefore vital. In [15] and [25] some measurement selection strategies are discussed, but no general conclusions could be drawn so far.

The novel method here proposed targets to minimize the influence of problem 1 and 2 .

\section{Methodology}

In this section the methodology of the new filtering method is described. First the principle and novelty of the method is explained. Afterwards, the different steps in the procedure are explained more in detail.

\subsection{Method}

The novelty of the new approach lies primarily in the way it estimates the modal parameters from the FRF matrix and how it subsequently corrects for frequency shifts. The new filtering method starts with estimating the modal parameters of each of the columns of the FRF matrix of assembly and test 
rig separately. As the individual FRFs in each of the columns are measured simultaneously, they are considered to contain the same poles. However, this is not necessarily true for the columns mutually, e.g. due to mass loading, changes in boundary conditions, etc. This is shown in figure 2 and further elaborated on in [23].

Subsequently the eigenfrequencies of the different columns of the FRF matrix are sorted and equalized. The other modal parameters are kept unchanged. This way inconsistencies in the FRF matrix, i.e. frequency shifts, are removed. It is assumed that the mode shapes are not significantly altered when moderate mass loading, or a change in boundary conditions is present. This is empirically shown later in the results, see figure 10.

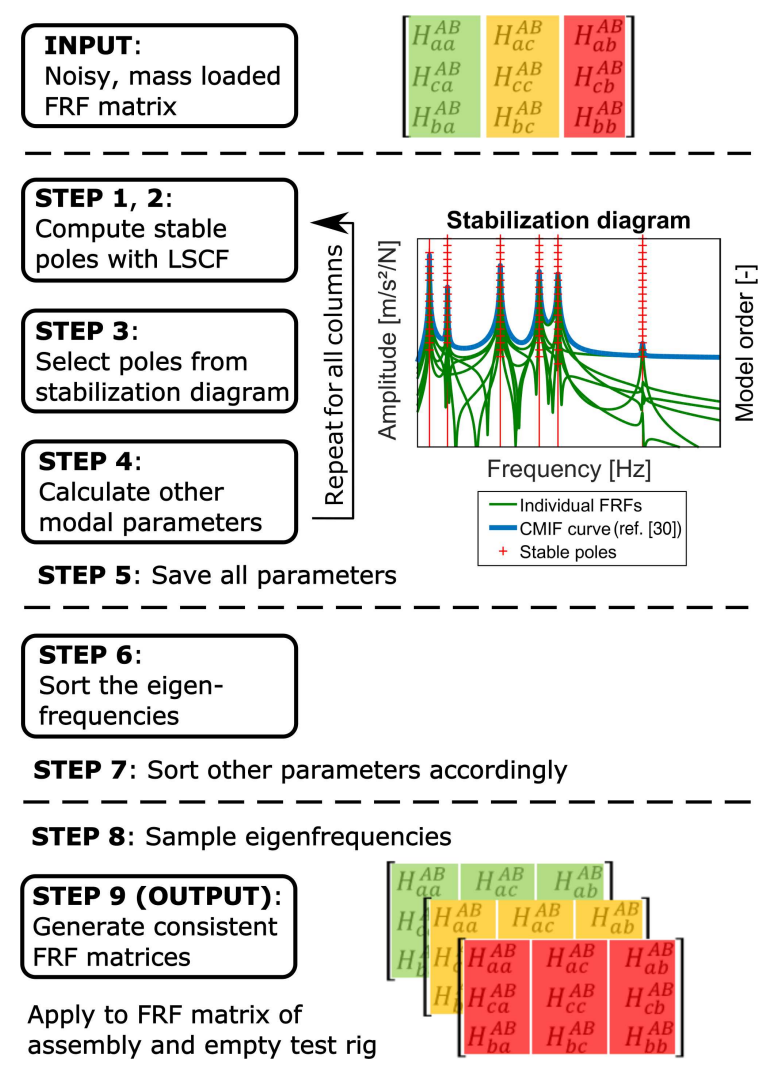

Figure 2: Workflow of the new filter 
By sampling the eigenfrequencies from the range in which they are measured, an arbitrary amount of consistent FRF matrices can be generated, while the uncertainty on the eigenfrequencies is taken into account. This differs from other filtering approaches that only generate a single filtered FRF matrix. No assumptions are made a priori to which of the observed eigenfrequencies are correct, therefore a uniform distribution is used for the generation eigenfrequencies. The method is applied on the FRF matrix of both the assembly and empty test rig. The workflow of the new filtering approach is visualized in figure 2 .

Afterwards all possible combinations of the FRF sets are used for the decoupling calculation. As a result, a large number of possible decoupling solutions is obtained. In the spread of these results, the sensitivity of the decoupling method can be observed, while for further processing the average or -assuming a normal distribution- upper $90 \%$ can be used. 


\begin{tabular}{|c|c|}
\hline \multicolumn{2}{|c|}{ Algorithm 1: Filtering algorithm (part 1) } \\
\hline \multicolumn{2}{|l|}{ NOTATION: } \\
\hline $\boldsymbol{w}=$ Eigenfrequencies & ${ }_{i}=$ Input index \\
\hline$z=$ Damping values & $s=$ Random sample \\
\hline $\boldsymbol{L}=$ Participation factors & $N_{i}=$ Nr. of inputs \\
\hline $\boldsymbol{L} \boldsymbol{R}, \boldsymbol{U} \boldsymbol{R}=$ Lower, upper residuals & $N_{g}=$ Nr. of generated matrices \\
\hline $\boldsymbol{\lambda}=$ Poles & $N_{p}=$ Nr. of poles \\
\hline $\boldsymbol{V}=$ Modeshapes & $\mathcal{U}=$ Uniform distribution \\
\hline $\boldsymbol{M}=$ Reduced matrix & $\bar{x}=$ Unsorted instances of $\mathrm{x}$ \\
\hline$\omega=$ Radial frequency & $\tilde{x}=$ Sorted instances of $\mathrm{x}$ \\
\hline \multicolumn{2}{|l|}{ INPUT: } \\
\hline \multicolumn{2}{|c|}{$\boldsymbol{H}=$ Complete FRF matrix of assembly or empty test rig } \\
\hline \multicolumn{2}{|c|}{$\boldsymbol{F}=$ Frequency axis } \\
\hline \multicolumn{2}{|c|}{$n=$ Maximum order of the polynomial model } \\
\hline \multicolumn{2}{|c|}{ OUTPUT: } \\
\hline$\widehat{\mathbf{H}}=$ Filtered, consistent FRF matric & \\
\hline
\end{tabular}


PART 1: ESTIMATING THE MODAL PARAMETERS

for $i=1, \ldots, N_{i}$ do

STEP 1: Calculate reduced matrix M with LSCF algorithm [26]

$$
\boldsymbol{M}=\operatorname{LSCF}\left(\boldsymbol{H}_{i}, n\right)
$$

STEP 2: Compute the stable poles for increasing model orders [26]

$$
\overline{\boldsymbol{\lambda}}_{1: n}=\operatorname{STABLE}(\boldsymbol{M}, \boldsymbol{F}, n)
$$

STEP 3: Modified Automatic Model Parameter Selection [27, 28]

$$
\left[\boldsymbol{w}_{i}, \boldsymbol{z}_{i}, \boldsymbol{L}_{i}\right]=\operatorname{AMPS}\left(\overline{\boldsymbol{\lambda}}_{1: n}\right)
$$

STEP 4: Modes, residuals are estimated in a least-squares way [26]

$$
\left[\boldsymbol{V}_{i}, \boldsymbol{L} \boldsymbol{R}_{i}, \boldsymbol{U} \boldsymbol{R}_{i}\right]=\operatorname{LSFD}\left(\boldsymbol{w}_{i}, \boldsymbol{z}_{i}, \boldsymbol{L}_{i}\right)
$$

end

STEP 5: Combine the parameters for all inputs.

$\overline{\boldsymbol{w}}=\boldsymbol{w}_{i} \quad \forall i=\left\{1, \ldots, N_{i}\right\}$

Identical action for $\boldsymbol{z}_{i}, \boldsymbol{L}_{i}, \boldsymbol{V}_{i}, \boldsymbol{L} \boldsymbol{R}_{i}$ and $\boldsymbol{U} \boldsymbol{R}_{i}$

\section{PART 2: SORTING THE EIGENFREQUENCIES}

STEP 6: Sort the eigenfrequencies based on frequency difference

OR MAC-values and frequency difference

$$
\widetilde{\boldsymbol{w}} \leftarrow \overline{\boldsymbol{w}}
$$

STEP 7: Sort all other parameters accordingly

$$
\widetilde{\boldsymbol{V}} \leftarrow \overline{\boldsymbol{V}} ; \widetilde{\boldsymbol{L}} \leftarrow \overline{\boldsymbol{L}} ; \widetilde{\boldsymbol{z}} \leftarrow \overline{\boldsymbol{z}}
$$

Note: empty elements are padded with zeros

\section{PART 3: GENERATING CONSISTENT FRF MATRICES}

for $u=1, \ldots, N_{g}$ do

STEP 8: Sampling the eigenfrequencies

$$
\boldsymbol{w}_{s}=\boldsymbol{w}_{r} \in \mathcal{U}\left(\widetilde{\boldsymbol{w}}_{r}^{\min }, \widetilde{\boldsymbol{w}}_{r}^{\max }\right) \forall r=\left\{1, \ldots, N_{p}\right\}
$$

STEP 9: Reconstructing the consistent FRF matrices [29]

$$
\widehat{\boldsymbol{H}}_{u}(j \omega)=\sum_{r=1}^{N_{p}}\left(\frac{\widetilde{\boldsymbol{V}}_{r} \boldsymbol{L}_{r}}{j \omega-\lambda_{r}}+\frac{\widetilde{\boldsymbol{V}}_{r}^{*} \boldsymbol{L}_{r}^{*}}{j \omega-\lambda_{r}^{*}}\right)-[\boldsymbol{U} \boldsymbol{R}] \omega^{2}+[\boldsymbol{L R}]
$$

With $\lambda_{r}=z_{r}+j w_{s}$

end 


\subsection{Workflow}

The workflow of the new approach is further explained in algorithm 1. The most important steps are described more in detail in the following paragraphs.

\subsubsection{Estimation of the modal parameters}

In this work, the estimation of the modal model from experimental data is done in two main steps as described by Van der Auweraer in [26]. First a common denominator polynomial model is fitted on the FRFs for increasing model orders. Then the poles are selected in a stabilization diagram and the damping and residues are estimated. This step is called the Least Squares Complex Frequency domain (LSCF) method. During a second step, the mode shapes and residuals are estimated with a linear least-squares frequency-domain (LSFD) solver [26]. Note that for single-input-multiple-output (SIMO) FRFs, identical results are obtained with the pLSCF algorithm [19, 20].

Because of the considerable amount of processing required to identify all columns of both the assembly and empty test rig separately, the automatic modal parameter selection procedure $[27,28]$ is used. This algorithm compares frequency and damping stability over a given set of model orders. In [27] it was reported that the algorithm tends to overestimate the number of stable poles (thus selecting also numerical poles). To overcome this, a singular value decomposition of the FRF column is used to plot the Complex Mode Indicator Function (CMIF) [30]. As the peaks detected in the CMIF plot indicate the existence of eigenfrequencies, a peak finding algorithm can reduce the frequency range in which eigenfrequencies can be detected. This way, the number of falsely identified poles is greatly reduced, while the number of unidentified physical poles is also very limited. Another advantage of the SIMO approach is that clear stabilization diagrams are obtained, despite possible mass loading [23].

It should be noted, as also indicated in [27], that no automatic methods exist for modal parameter selection that work in all scenarios. However, the described method proved to be successful on both numerical and experimental data if SIMO FRFs are used, with relatively high signal-to-noise ratios. A 
limited amount of manual user interaction is however still required to verify that no error in the automatic process occurred. Now that all poles are identified, the remaining modal parameters (mode shapes and upper and lower residuals) are identified.

\subsubsection{Sorting of the eigenfrequencies}

Afterwards, the different poles must be sorted before the FRFs can be made consistent. As some poles may be absent in certain FRFs when the excitation is located on a node, some sorting is needed. This can be done in two ways, based on the eigenfrequencies, or both the eigenfrequencies and modeshapes.

If the distance between the eigenfrequencies is significantly larger than the variation on the eigenfrequencies, a simple algorithm can be written that sorts the poles (and corresponding modal parameters) on the basis of frequency difference.

If the modal density is of the same order of magnitude as the variations in eigenfrequencies, the sorting of the poles may be difficult on the basis of frequency difference alone. In this case, also the modeshape can be compared using a MAC diagram. This requires a sufficient amount of sensors to fully capture and distinguish the eigenmodes, especially at higher frequencies [31]. It should also be noted that some modes may be badly excited if the excitation is close to a nodal point. In this case, the mode may be badly, or not estimated. This can make the sorting of the modes difficult.

The choice of sorting algorithm is up to the user. In this work good results were obtained when the algorithm based on the frequency difference is used for hammer measurements and cases with very little mass loading. When moderate or heavy mass loading is present, the sorting algorithm that also takes into account the modes, is the most reliable.

\subsubsection{Generation of consistent FRF matrix matrices}

The FRF matrices are reconstructed using equation 8. Each FRF is reconstructed with its original modeshapes, participation factors, damping and 
residuals. The eigenfrequencies are sampled from the range in which they are measured. It is also possible to manually define a range in which the poles are considered to be located. This may be useful when the final design of a component is not yet finished or when the boundary conditions are uncertain. It can also reduce the effect of modelling errors as they are averaged out.

Since it is not known which of the solution is the best or most accurate, an arbitrary amount of consistent FRF matrices is generated. The amount is mainly limited by memory requirements and calculation time. Finally, all possible combinations of assembly and empty test rig matrices are used in equation 7 , resulting in many decoupling solutions.

The average solution can be used for further processing or - assuming a normal distribution - the upper upper $90 \%$. The spread on the results shows the sensitivity or reliability of the decoupling method.

\section{Results}

The new method will be tested on both numerical and experimental cases. It will be compared with the more standard approach of using raw FRFs. Both methods are used in combination with a TSVD. The numerical FRFs will be polluted with different types of noise. This way, it will be demonstrated what type of error is the most detrimental for the quality of decoupled FRFs for both methods. For the experimental cases, both hammer and shaker measurements will be shown.

In order to have a more objective criterion for the comparison of the FRFs, the frequency assurance criterion (FRAC) [31] will be used to compare the decoupled FRFs with the reference solution. The reference solution is obtained by calculating or measuring the FRFs of the decoupled structure directly. The FRAC between FRF A and B is calculated as follows:

$$
\boldsymbol{F} \boldsymbol{R} \boldsymbol{A} \boldsymbol{C}^{A B}=\frac{\left|\boldsymbol{H}^{A}(\omega) \boldsymbol{H}^{B}(\omega)^{T}\right|^{2}}{\left(\boldsymbol{H}^{A}(\omega) \boldsymbol{H}^{A}(\omega)^{T}\right) *\left(\boldsymbol{H}^{B}(\omega) \boldsymbol{H}^{B}(\omega)^{T}\right)}
$$


In [31] it is stated that FRAC values can sometimes be quite low while visually the correlation looks good. This is attributed to errors in the phase. In this case it can be useful to use the absolute values of the FRFs.

As stated earlier, both the new and the standard approach make use of a TSVD for the inversion. Depending on the theoretical number of connection DoFs, noise and other errors, the SVD is truncated at a certain amount of singular values. If too few singular values are retained, the peaks of the decoupled result will be badly estimated (the damping is overestimated). If too many are retained, the results may become very noisy and spurious peaks, due to e.g. curve fitting errors, become important. In the theoretical case of perfect FRFs, the extended interface method (using all available measurement locations) is singular at all frequencies and requires as many singular values as there are connection DoFs [11]. Since the modal model acts as a filter to noise, it can generally use more singular values compared to the standard approach before the noise is amplified too strongly. The choice of singular values may require some iteration if no reference solution is available. The influence of different singular values for the standard approach is illustrated in [21]. In this work, the number of singular values corresponding to the solution with the highest average FRAC value are used.

\subsection{Simulations}

The new approach will be tested on two numerical models. The first model is a $6-\mathrm{DoF}$ analytical lumped-mass model with 2 coupling DoFs. The second is an FE model of a test rig that will also be used for experimental decoupling. This test rig is decoupled in 6 DoFs. First the different types of noise that will be used to pollute the FRFs will be briefly explained.

\subsubsection{Noise modelling}

In order to assess the impact of noise and inconsistencies on the decoupled FRFs, the following types of noise are used:

- Background noise: 
A uniformly distributed random complex matrix is added to the FRF data. This mainly affects the amplitude and phase of the FRFs with the lowest magnitude, therefore simulating a noise floor on the FRF data.

- Multiplicative noise:

Each value in the FRF matrix is multiplied with $(1+R)$, with $R$ being a small zero-mean random complex value with a uniform distribution. In this case $\frac{1}{R^{2}}$ approximates the signal-to-noise ratio. It affects the whole FRF independently of the magnitude.

- Phase noise:

In this case, the phase of the FRF signal is perturbed with a zero-mean uniformly distributed random value without affecting the amplitude of the FRF. Phase noise is considered because the phase of driving point FRFs is a good indicator for the quality of the measurement. It can be shown that small errors in the driving point phase have a strong detrimental impact on the decoupling results.

- Mass loading:

To simulate mass loading, the elements of the (diagonal) mass matrix are multiplied by $1+\mathrm{R}$, with $\mathrm{R}$ being a zero-mean normally distributed random value. This is repeated for each column of the FRF matrix.

- Frequency shifting:

As the calculation of direct FRFs from large FE models is computationally expensive, frequency shifts are introduced on the columns of the FRF matrices of the FE model to approximate the effect of mass loading. This is done by simply shifting the FRFs of a single column along the frequency axis with a random number of frequency lines.

\subsubsection{Analytical model}

The first model is an analytical 6-DoF lumped mass model with 2 coupling degrees of freedom. The model can be decoupled at the locations of masses $m_{2}$ 
and $m_{3}$ as shown in figure 3 . The decoupled system therefore consists of masses $m_{2 b}, m_{3 b}$ and $m_{4}$. All parameters are listed in table 1. Complex damping is chosen at $0.5 \%$. Frequencies are considered between 20 and $400 \mathrm{~Hz}$.

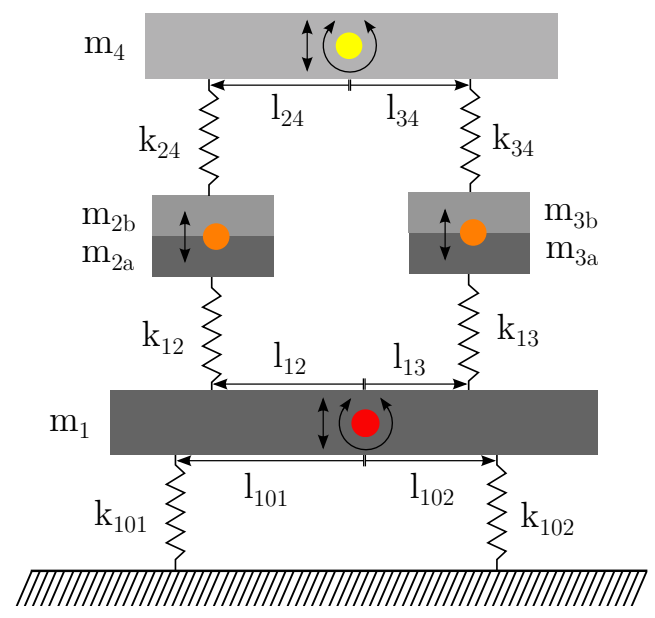

Figure 3: 6-DoF analytical model

As a first validation, the method is used on perfect unperturbed FRFs from the analytical model. The modal parameters are automatically estimated and sorted as described in the previous section. The individual solutions (blue) as well as the average (red) and the upper $90 \%$ (orange) solutions are plotted against the reference solution (green) in figure 5. The driving point FRF at Connection 2 (C 2) is considered. It is clear that the variation on the result is extremely small. In fact, the only source of uncertainty is in the way the different columns of the FRF matrix are treated by the modal estimation algorithms. This is a characteristic of the modal estimation procedure. Because of the low uncertainty, the number of calculations is limited to just 50 .

Indeed, with an average relative error of around $0.01 \%$, the decoupled FRFs are very accurate. The minimum value of the frequency response assurance criterion (FRAC) [31] is $99.6 \%$. This shows that the modal model filter can be accurate enough, at least when the condition number of the inversion is not very high. When perfect data is considered, the condition number for this model is 


\begin{tabular}{ll} 
Mass $[\mathrm{kg}]$ & \\
\hline$m_{1}=40$ & \\
$m_{4}=50$ & \\
$m_{2}=m_{2 a}+m_{2 b}$ & $=5+10=15$ \\
$m_{3}=m_{3 a}+m_{3 b}$ & $=11+7=18$ \\
\hline Inertia $[\mathrm{kgm}]$ & \\
\hline$l_{1}=2.5$ & \\
$l_{4}=3$ & \\
\hline Stiffness $[\mathrm{N} / \mathrm{m}]$ & \\
\hline$k_{101}=1.0 e 7$ & $k_{102}=1.5 e 7$ \\
$k_{12}=6.0 e 6$ & $k_{13}=7.0 e 6$ \\
$k_{24}=8.0 e 6$ & $k_{34}=7.0 e 6$ \\
\hline Length $[\mathrm{m}]$ & \\
\hline$l_{101}=0.5$ & $l_{13}=0.45$ \\
$l_{102}=0.5$ & $l_{24}=0.45$ \\
$l_{12}=0.45$ & $l_{34}=0.45$ \\
\hline
\end{tabular}

Table 1: Properties of the analytical model

31 on average with peaks into the several hundred when the extended interface method is used with 2 out of 4 singular values. All of the peaks can be traced back to the eigenfrequencies of one of the 3 subsystems. This is illustrated in figure 4 .

To test its filtering capabilities, the modal model will be used to filter different types of noise on the FRF matrices of the assembly and empty test rig. The number of decoupling calculations is set to a maximum of 500. The results will be compared with those from the more often used raw FRFs, both in combination with a TSVD. For both methods, the extended interface method for decoupling is used. For reasons of comparability, the same noise levels will be used as in [21]. The goal is to compare the boundaries where both methods provide meaningful results. 


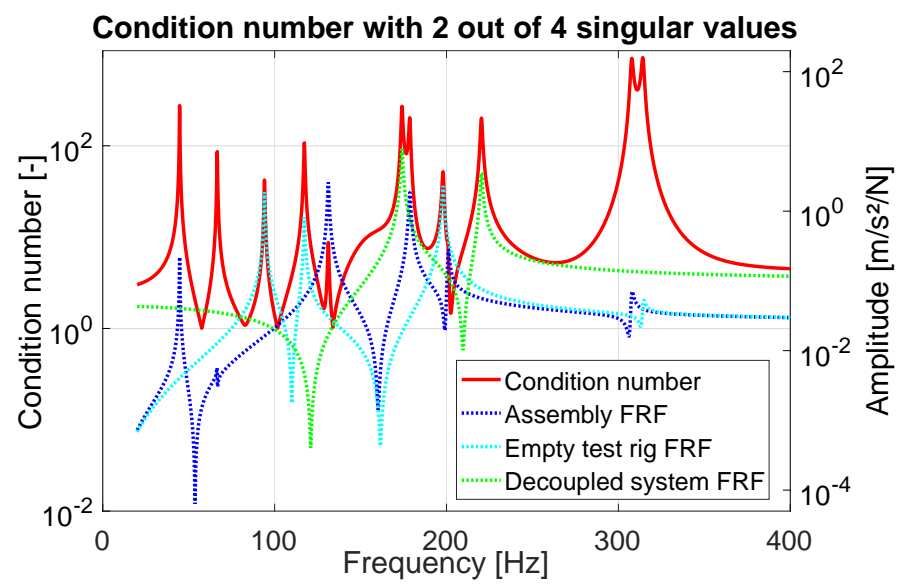

Figure 4: Condition number for the analytical model with 2 out of 4 singular values (extended interface method)

\begin{tabular}{lcc}
\hline FRAC & Modal model & Standard approach \\
\hline Minimum & 0.986 & 0.182 \\
Average & 0.994 & 0.410 \\
Maximum & 0.997 & 0.624 \\
\hline
\end{tabular}

Table 2: Analytical model with background noise: FRAC results

\section{Background noise.}

In the first case the FRFs are polluted with a background noise level of $5 e-3$ $\left[m / s^{2} / N\right]$. An example of a decoupled FRF for both methods is shown in figure 6. It is clear that the new method is insensitive to background noise compared to the standard approach, even when an SVD is used. Best results are obtained for both methods by using 3 and 2 (out of 4 ) singular values respectively. The corresponding FRAC values are listed in table 2.

Phase noise.

When introducing random errors of maximum 5 degrees in the phase of the FRF, the results deteriorate significantly. Especially the standard approach is very sensitive to phase errors, resulting in very noisy FRFs. The modal 

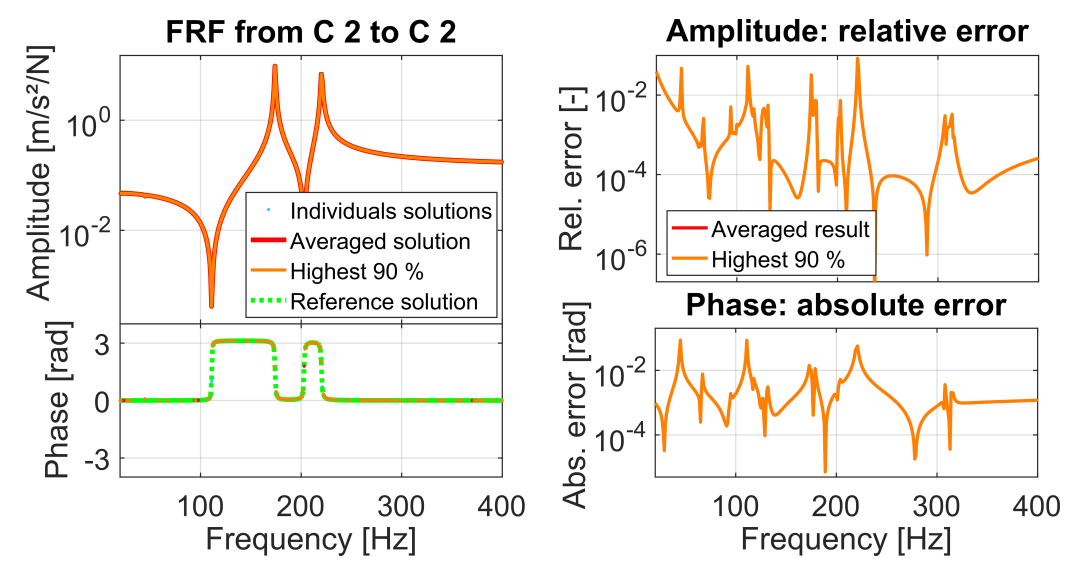

Figure 5: Example of a decoupled FRF using the modal model filter

\begin{tabular}{lcc}
\hline FRAC & Modal model & Standard approach \\
\hline Minimum & $3 \mathrm{e}-4(0.650)$ & $0.071(0.305)$ \\
Average & $0.070(0.746)$ & $0.225(0.452)$ \\
Maximum & $0.194(0.798)$ & $0.271(0.526)$ \\
\hline
\end{tabular}

Table 3: Analytical model with phase noise: FRAC results with complex (absolute) values

model also suffers from the phase errors in the FRF matrix resulting in spurious peaks in some FRFs. However, as these spurious peaks consistently appear at eigenfrequencies of one of the two original systems, they are easily filtered out in further processing. As an example, the decoupled FRF between the connections (C) is shown in figure 7 .

The minimum, average and maximum FRAC values of the decoupled FRFs for both methods, shown in table 3, are significantly lower than the previous case. More importantly, the FRAC values for the modal model are lower than those for the standard approach, while the FRF shown in figure 7 is significantly less noisy and easier to interpret. Therefore, the FRAC values are also calculated with the absolute values of the FRFs [31]. This is shown between brackets in table 3 . Indeed, the absolute FRAC values confirm that the modal model provides a better estimate of the decoupled FRFs, at least when only 


\section{Analytical model: FRF from C 1 to $C 1$ : new vs. standard approach}
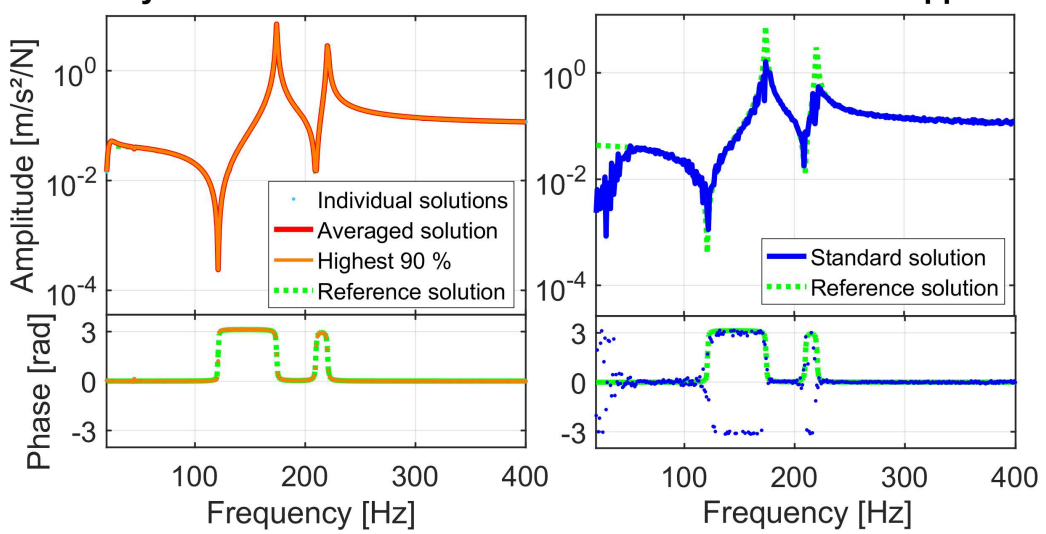

Figure 6: Analytical model: new approach (left, 3/4 sing. vals) vs. standard approach (right, $2 / 4$ sing. vals): background noise

\begin{tabular}{lcc}
\hline FRAC & Modal model & Standard approach \\
\hline Minimum & 0.867 & 0.268 \\
Average & 0.906 & 0.483 \\
Maximum & 0.968 & 0.694 \\
\hline
\end{tabular}

Table 4: Analytical model with multiplicative noise: FRAC results

the amplitude of the FRFs is considered. Some errors in the phase are indeed present, but these do not outweigh the noise present in the FRFs of the standard approach.

Multiplicative noise.

The effect of multiplicative noise is relatively limited: the FRAC values of the modal model approach are very high (see table 4) and both methods provide FRFs that are easily interpretable. The decoupled FRFs of the standard approach are noisy in the lower amplitudes whereas the modal model approach exhibits very little spurious peaks or systematic errors. An example is shown in figure 8.

Mass loading. 
Analytical model: FRF from C 2 to C 1: new vs. standard approach
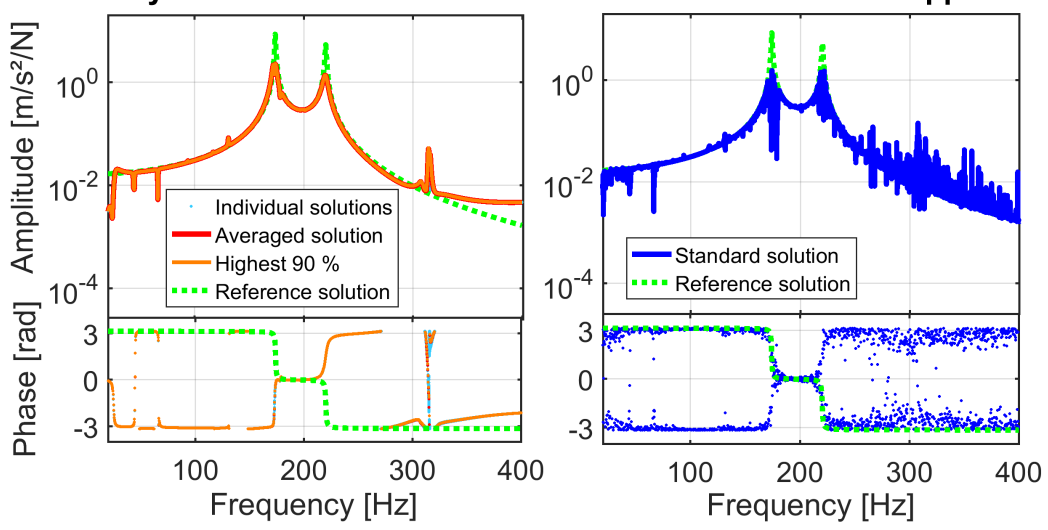

Figure 7: Analytical model: new approach (left, $3 / 4$ sing. vals) vs. standard approach (right, $3 / 4$ sing. vals): phase noise

To simulate the effect of mass loading, each column of the FRF matrix of the assembly and empty test rig is calculated with a mass matrix of which the (diagonal) elements are perturbed with a normal random distribution with a value of $5 \%$ of the original mass as standard deviation. The effect is a shift of the eigenfrequencies that increases with frequency. The variation on each of the eigenfrequencies of the assembly is shown in figure 9. The diagonal of a MAC diagram between the estimated modes from two different columns of the FRF matrix of the assembly confirms the assumption that the modes shapes are not significantly altered. This is shown in figure 10.

The effect on the decoupled FRF is most significant at the higher frequencies where a clear spurious peak is observed around $310 \mathrm{~Hz}$ in some of the FRFs in the standard approach. As there is some significant uncertainty on the eigenfrequencies, the use of the new approach is best illustrated. By taking many averages, the modal model is capable of reducing this peak and indicating that this area is highly uncertain. Increasing the number of decoupling calculations can further average out this peak. The standard approach suffers from significant spurious peaks, especially around $300 \mathrm{~Hz}$, where the $6^{\text {th }}$ mode is situated. 


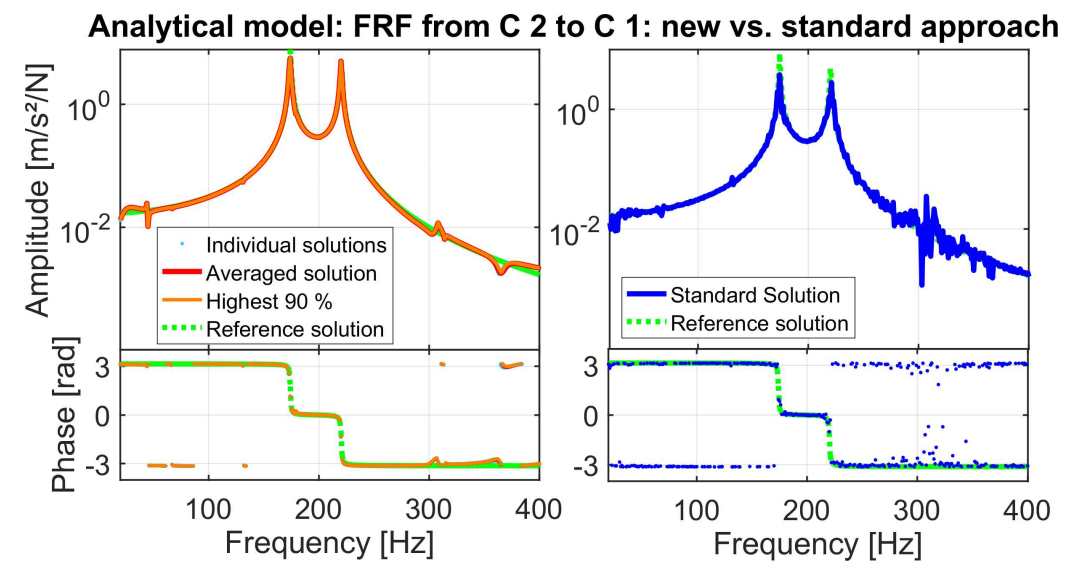

Figure 8: Analytical model: new approach (left, $3 / 4$ sing. vals) vs. standard approach (right, $3 / 4$ sing. vals): multiplicative noise

\begin{tabular}{lcc}
\hline FRAC & Modal model & Standard approach \\
\hline Minimum & 0.727 & 0.353 \\
Average & 0.862 & 0.696 \\
Maximum & 0.915 & 0.945 \\
\hline
\end{tabular}

Table 5: Analytical model with mass loading: FRAC results

The right column in table 5 shows that by using more singular values ( 3 instead of 2), the standard approach can still provide good results for the least sensitive FRFs. The modal model on the other hand shows an improvement for the most sensitive FRFs as well as on average while losing only little accuracy on the least sensitive FRFs.

\section{Combination of noise.}

As real measurements generally exhibit a combination of different types of noise and/or mass loading, the noise values from the cases above are combined. An example of a decoupled FRF is shown in figure 12. In this scenario, the model modal approach has the most use. It filters the noise and makes the columns of the FRF matrices more consistent with regard to each other. While none of the individual solutions are perfect (blue dots), the averaged result (red curve) 


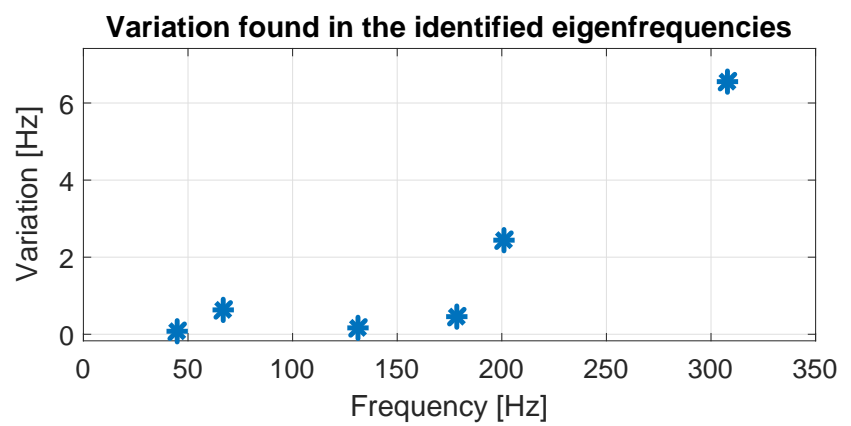

Figure 9: Mass loading: uncertainty on the estimated eigenfrequencies

or upper $90 \%$ curve (orange curve) approximates the solution well, while only small spurious peaks are still visible. The results are confirmed by the FRAC values of the complex and absolute values of the FRFs for both approaches. This is shown in table 6 .

\begin{tabular}{lcc}
\hline FRAC & Modal model & Standard approach \\
\hline Minimum & $0.111(0.603)$ & $0.023(0.175)$ \\
Average & $0.320(0.653)$ & $0.089(0.230)$ \\
Maximum & $0.505(0.680)$ & $0.131(0.299)$ \\
\hline
\end{tabular}

Table 6: Analytical model with combined noise: FRAC results with complex (absolute) values

\subsubsection{FE model}

The second example is an FE model of a test rig that will also be used for experimental decoupling. It is shown in figure 13. The design of the test rig is similar to a test rig presented by D'Ambrogio and Fregolent in [16, 17], although some slight modifications are introduced. The design was chosen because a relatively good decoupling was obtained [17] while leaving room for improvement. The test rig consists of 4 aluminium beams with a cross section of $50 \times 10 \mathrm{~mm}$ connected with 4 M6 bolts at each of the connections. The upper horizontal 


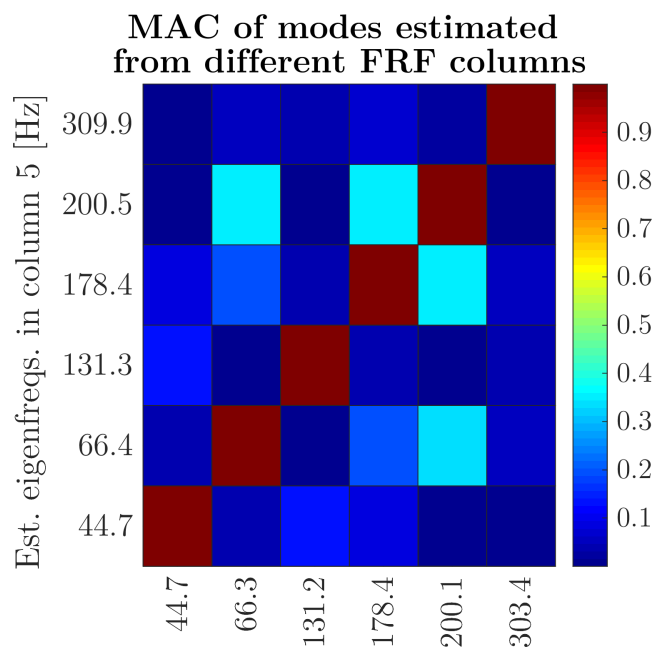

Est. eigenfreqs. in column $3[\mathrm{~Hz}]$

Figure 10: Mass loading: influence on the modes

beam will be decoupled from the lower structure using the extended interface method. The square aluminium piece at the right of the upper beam is added to ensure the first 5 eigenmodes are below $1 \mathrm{kHz}$ and thus easily excitable by a hammer with soft tip. As a consequence, the first and second in-plane mode of the decoupled upper beam are also observed in the out-of-plane direction. A list of the dimensions is shown in table 7 . The structure is suspended very softly using bungees to approximate free-free conditions. The black plastic straps in figure 13 are removed during testing.

\begin{tabular}{cccccccc}
\hline Dimension & $L_{1}$ & $L_{2}$ & $L_{3}$ & $L_{4}$ & $L_{5}$ & $L_{6}$ & $L_{7}$ \\
{$[\mathrm{~mm}]$} & 360 & 80 & 30 & 550 & 200 & 100 & 250 \\
\hline
\end{tabular}

Table 7: Test rig dimensions

The FE model consists of 532 quadratic shell elements and 1911 nodes. This ensures at least 20 elements along the length of the wavelength of all modes encountered up to $4.5 \mathrm{kHz}$. The material properties are taken from standard tables. The density is $2700 \mathrm{~kg} / \mathrm{m}^{3}$ and the stiffness is $72 \mathrm{GPa}$. The connections 

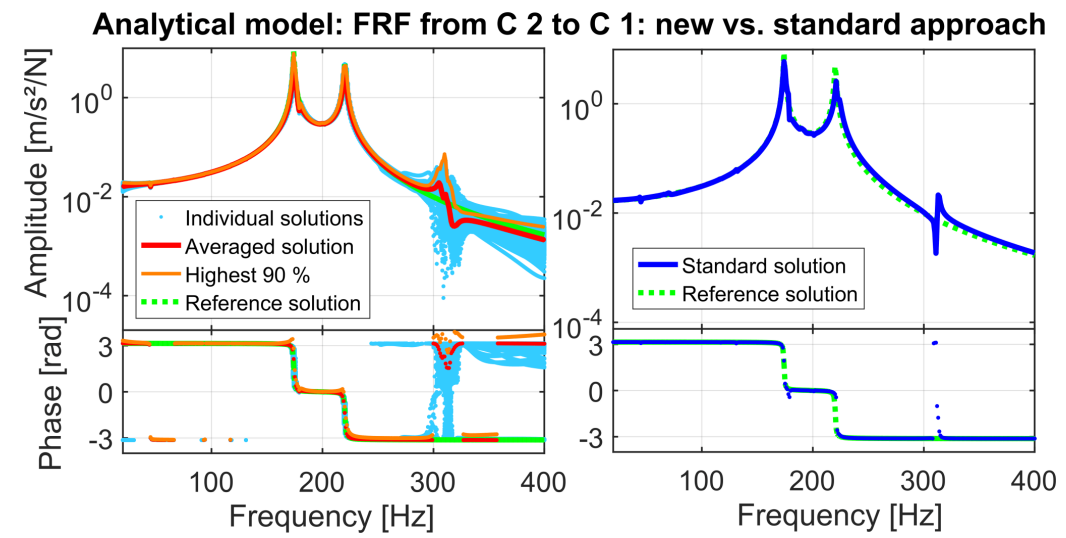

Figure 11: Analytical model: new approach (left, $3 / 4$ sing. vals) vs. standard approach (right, $3 / 4$ sing. vals): mass loading

are made with rigid 6-DoF multi-point constraint (MPC) elements. The model is considered in free-free conditions. The proposed method is tested first on clean FRFs obtained with a direct frequency response analysis. The excitation takes place at the nodal locations in the FE model. Afterwards the same procedure will be followed as for the previous example. Only the out-of-plane motion is considered. The information of the out-of-plane direction at the connection is used for decoupling, augmented with 25 DoFs distributed over the test rig. The reference solution of the FE model is calculated by performing the direct frequency response analysis on the beam alone.

Clean FRFs. As the FE model is significantly more complex than the analytical model and the 6-DoF connection is modelled as perfectly clamped (RBE2), the condition of the inversion is higher. Whereas the analytical model has an average condition number of just 32 with perfect FRFs (the extended interface method was used), the FRF matrix of the FE model has an average condition number of $1.2 \mathrm{e} 7$ with peaks up to $1 \mathrm{e} 9$. It is therefore likely that any modelling or measurement errors will be strongly amplified. To counter some of the effects of the modelling errors on the decoupled FRFs, a perturbation of $10 \mathrm{~Hz}$ on the eigenfrequencies and 1000 decoupling calculations are used on clean FRFs. 12 


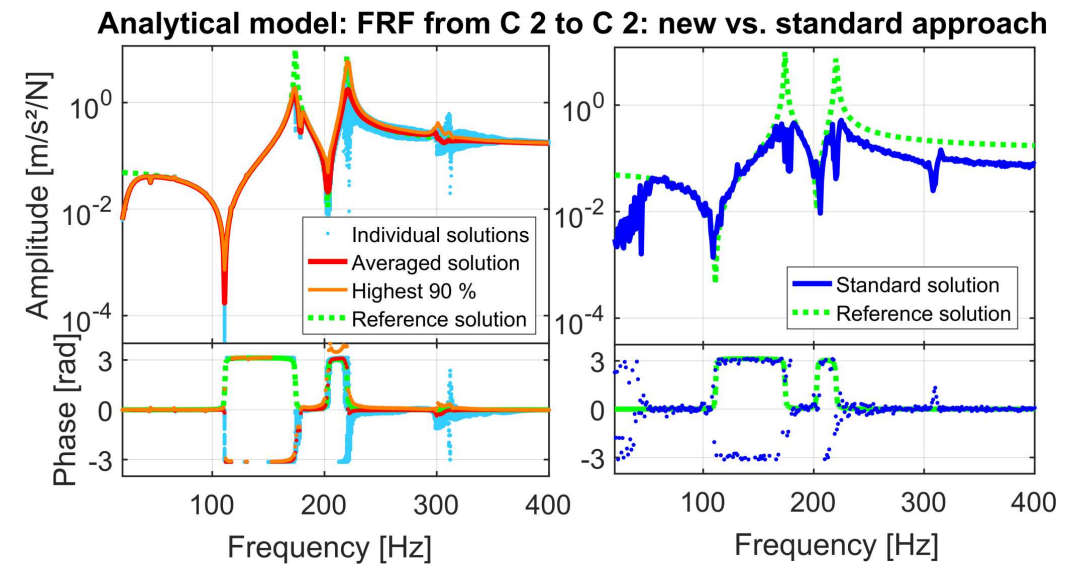

Figure 12: Analytical model: new approach (left, $3 / 4$ sing. vals) vs. standard approach (right, $2 / 4$ sing. vals): combination of noise

\begin{tabular}{ll}
\hline Type of noise & Value \\
Background noise & $2 e^{-2} \mathrm{~m} / \mathrm{s}^{2} / \mathrm{N}$ \\
Multiplicative noise & $2 e^{-2} \mathrm{~m} / \mathrm{s}^{2} / \mathrm{N}$ \\
Phase noise & $1^{\circ}$ \\
Frequency shifts & Max. $8 \mathrm{~Hz}$ \\
\hline
\end{tabular}

Table 8: Noise pollution values for FE model

singular values were used for the inversion of the modal model and 6 for the standard decoupling method.

Polluted FRFs. In order to test the resistance to noise, a combination of different types of noise is added to the FRFs. The noise values are listed in table 8. In the FRF shown in figure 15, there is a clear improvement around the eigenfrequency at $1600 \mathrm{~Hz}$. It is clear that that modal filter is much less sensitive to noise and inconsistencies than the standard approach. This is confirmed by the FRAC-values. 


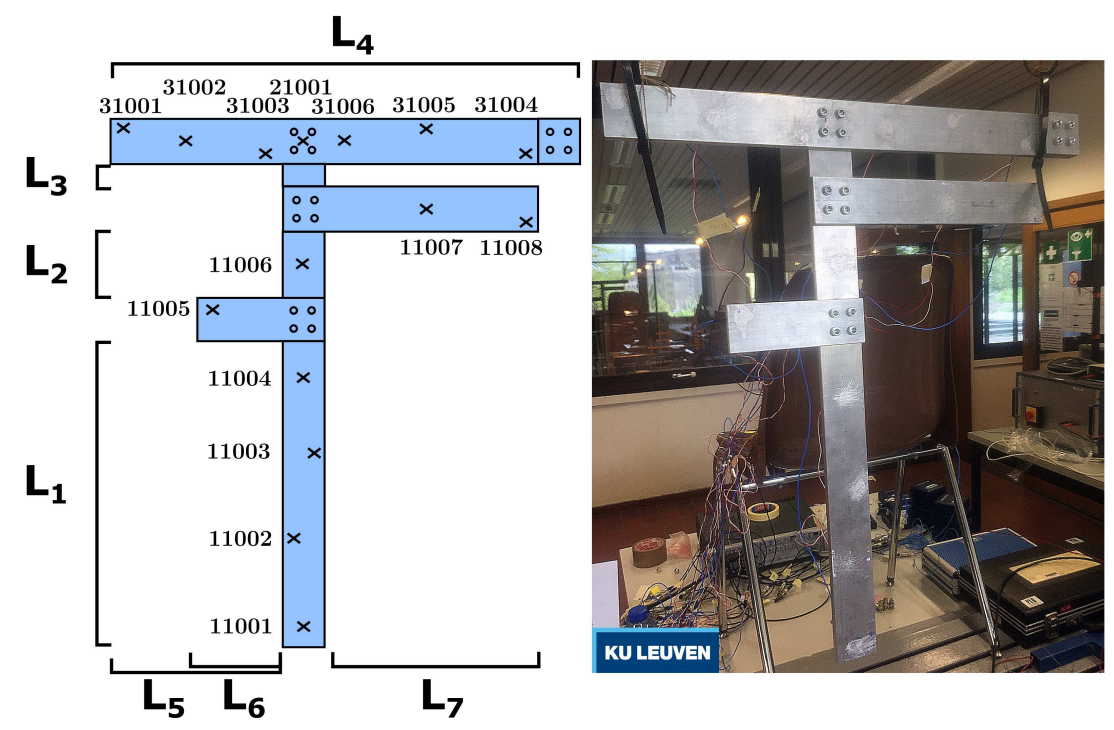

Figure 13: Design with measurement locations and photo of test rig

\begin{tabular}{lccc}
\hline FRAC & $\begin{array}{c}\text { Modal model } \\
\text { Clean }\end{array}$ & $\begin{array}{c}\text { Modal model } \\
\text { Noisy }\end{array}$ & $\begin{array}{c}\text { Standard approach } \\
\text { Noisy }\end{array}$ \\
\hline Min. & 0.335 & 0.243 & 0.052 \\
Average & 0.687 & 0.529 & 0.160 \\
Max. & 0.873 & 0.686 & 0.297 \\
\hline
\end{tabular}

Table 9: FRAC-values for the FE model FRFs

\subsection{Experimental results}

The test rig presented in the previous section was built and will be decoupled using shaker measurements as well as hammer measurements. The 15 collocated excitation and response locations are shown in figure 13.

\subsubsection{Shaker measurement}

During the measurements, a shaker is placed at different locations in order to obtain different columns of the FRF matrices. The structure is suspended using elastic bands on a fixed frame. As the structure is relatively light in comparison to the weight of the shaker, the mass loading is considerable, especially at high 


\section{FE model: a decoupled FRF: new vs. standard approach}
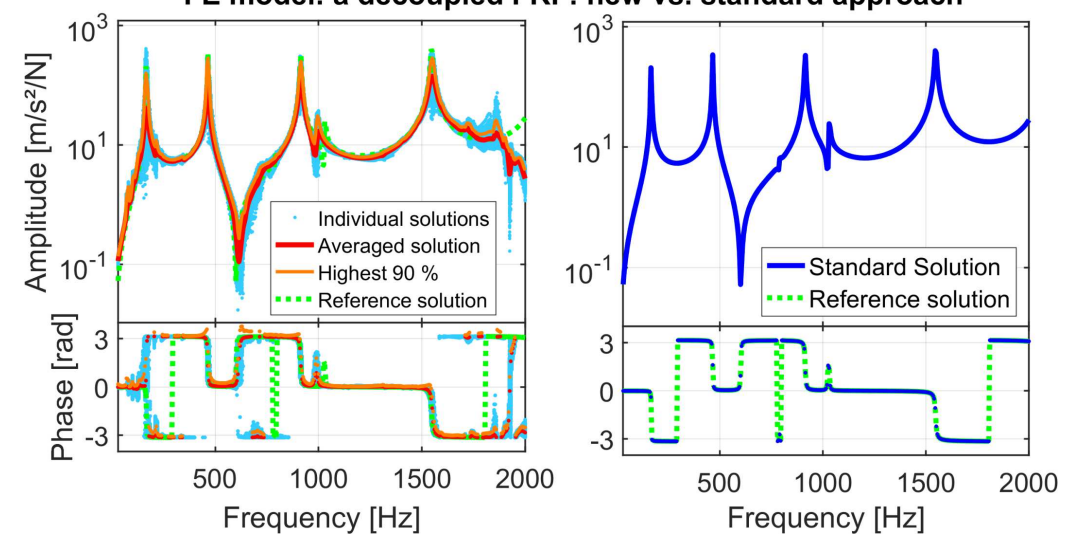

Figure 14: FE model: new approach (left, 12/26 sing. vals) vs. standard approach (right, 6/26 sing. vals): decoupled FRF with clean data

frequencies. To reduce some of this effect, some masses are glued to the structure at the locations at which the shaker is currently not exciting. As expected, the decoupling results are better at the lower frequencies. However, the goal of this paper is not to determine the best decoupling strategy for this particular structure, but to show that inconsistent measurements can be improved by a modal model.

The identification of the modes is done with the modified automatic modal parameter selection procedure. The modes are subsequently sorted based on a MAC analysis. A matrix of the sorting of the modes and the variation of the corresponding eigenfrequencies is shown in figure 16. All modes (Y-axis) are listed for each measurement run (X-axis) and the frequency has been given a color for easy interpretation. It is clear that most, but not all modes are consistently identified in the different measurement runs. This is not necessarily a problem for the method, but it reduces the possible gains as the FRF matrix is not fully consistent. For example, around $600 \mathrm{~Hz}$, there are several closely spaced modes which tend to be strongly influenced by the excitation position, causing them to switch order or disappear in some excitation points (based on the MAC analysis). This makes it hard to identify and subsequently sort them. 
FE model with noise: a decoupled FRF: new vs. standard approach
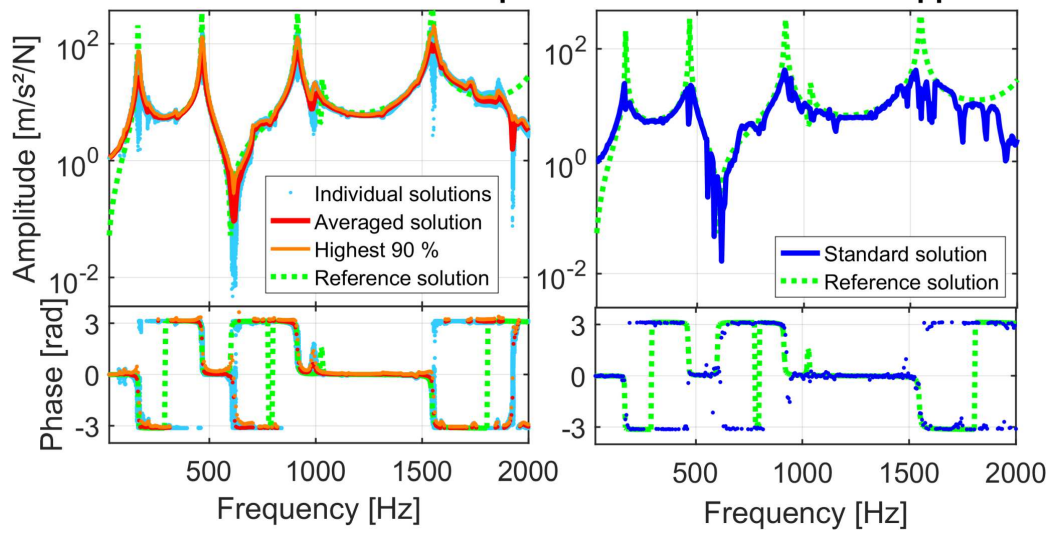

Figure 15: FE model: new approach (left, 12/26 sing. vals) vs. standard approach (right, 9/26 sing. vals): decoupled FRF with noisy data

The difference between highest and lowest identified eigenfrequency of identical modes is shown in figure 16 on the right. The variation is significant and follows roughly the same trend as the mass loaded 6-DoF model shown in figure 9. The reconstructed FRFs contain eigenfrequencies sampled from this variation. An example of different versions of a reconstructed FRF is shown in figure 17.

Figure 18 shows an example of a decoupled FRF calculated with both methods. It is clear from the left plot that a lot of uncertainty exists in the higher frequencies as the spread of the individual decoupling solutions is quite large (blue dots). With the standard approach, shown in the right plot, the $3^{\text {rd }}$ bending mode around $750 \mathrm{~Hz}$ is not visible in the decoupled FRF and some spurious peaks are present, e.g. at $320 \mathrm{~Hz}$. The modal model filter is better capable of reconstructing the two modes at 750 and $800 \mathrm{~Hz}$ while it shows a more stable FRF in the area between the eigenfrequencies. Also the first eigenfrequency is better reconstructed with the modal model filter, although the spurious peak around $150 \mathrm{~Hz}$ is present for both methods.

The FRAC values calculated with complex (absolute) values are shown in table 10. The average and maximum FRAC values (both for complex and abso- 

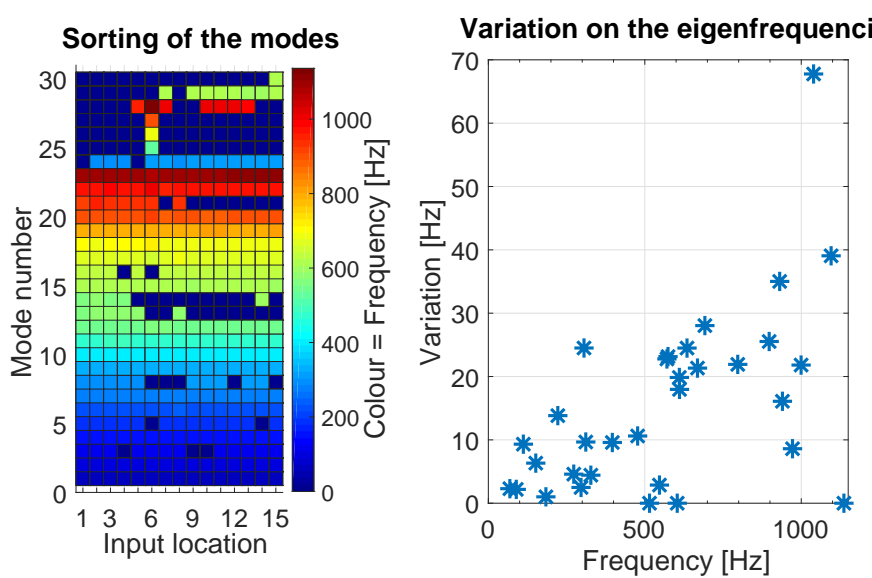

Figure 16: Shaker measurement: sorting and variation of the eigenfrequencies

lute values) are higher with the new approach. This confirms the improvement over the standard approach. The applied modal model provided an improve-

\begin{tabular}{lcc}
\hline FRAC & Modal model & Standard approach \\
\hline Minimum & $0.001(0.106)$ & $0.002(0.006)$ \\
Average & $0.032(0.237)$ & $0.022(0.135)$ \\
Maximum & $0.121(0.532)$ & $0.069(0.390)$ \\
\hline
\end{tabular}

Table 10: Shaker measurements: FRAC results with complex (absolute) values

ment for most decoupled FRFs but suffered from spurious peaks and systematic errors in other FRFs. In these FRFs it did not bring a substantial improvement compared to the standard method, other than showing a large spread on the results, indicating a large amount of inconsistency. It was observed in both experimental and numerical experiments, that FRFs between lowly coupled directions (lower in amplitude) suffer the most from experimental and modelling errors. 


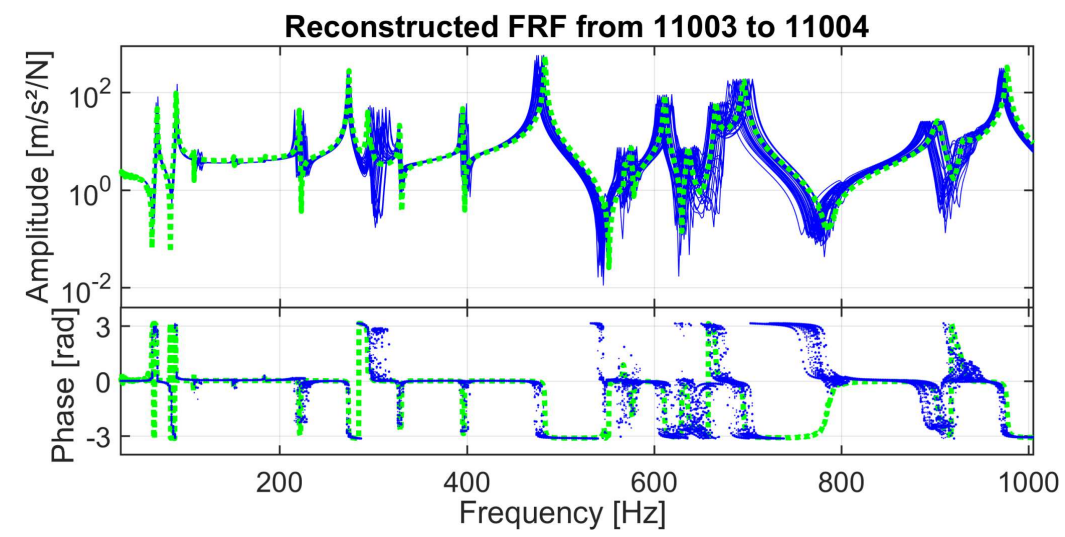

Figure 17: Different versions of a reconstructed FRF

\subsubsection{Hammer measurement}

In the previous section, the method was tested on a rather extreme case of mass loading. To test how the method performs with good measurement data, the structure was excited with a hammer with aluminium tip in the same points as shown in figure 13. The sorted poles and the maximum difference found for the eigenfrequencies is shown in figure 19. It is clear that the consistency of the data is much higher. Almost all modes are consistently identified and the variation in the eigenfrequencies is limited to less than $3 \mathrm{~Hz}$ in the frequency range below $1 \mathrm{kHz}$. The small variation that is still present in the eigenfrequencies is attributed to minor changes in the location of the suspension during the measurement. Similar consistency was observed in the FRF matrix of the empty test rig.

Indeed, with such good data, the decoupling results of both methods are very good. 7 out of 9 singular values are used for both methods. The eigenfrequencies of the modal model are perturbed with a maximum of $10 \mathrm{~Hz}$ and 2500 decoupling calculations are performed. An example of a decoupled FRF is shown in figure 20. Both the averaged and upper $90 \%$ curves provide a very good estimate of the decoupled FRF.

It should be noted that whereas most FRFs are very well reconstructed, some 


\section{Experiments: FRF from 31006 to 31001 : new vs. standard approach}
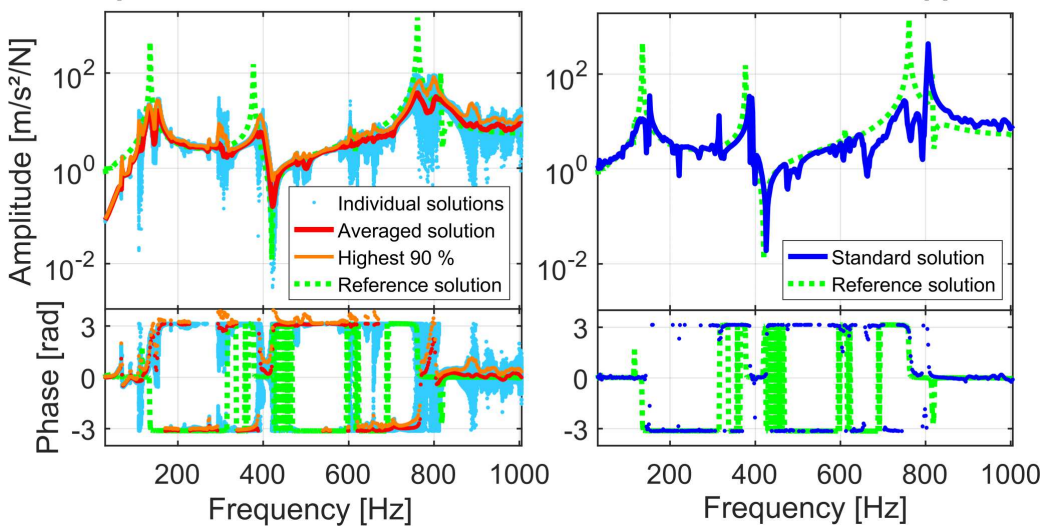

Figure 18: Shaker excitation: new approach (left, 9/9 sing. vals) vs. standard approach (right, $7 / 9$ sing. vals)

FRFs remain very sensitive to noise and modelling errors for both methods. An example of such an FRF is shown in figure 21. In some FRFs a consistent spurious peak at $650 \mathrm{~Hz}$ is present in both methods.

\section{Conclusions and future work}

\subsection{Conclusions}

In this paper a new method for decoupling is proposed that is able to filter the noise from FRF matrices and quantify and correct inconsistencies. The method is particularly useful when the highest quality of FRF measurements for experimental decoupling cannot be guaranteed. The new method filters the experimental data by estimating the modal parameters for each column of the FRF matrix separately. An existing algorithm for automatic modal parameter selection (AMPS) has been augmented with an extra filter based on the CMIF plot of the FRF column, facilitating the AMPS. Then, the eigenfrequencies and modes are compared and sorted based on the frequency difference or MAC-value. Based on the observed variation of the eigenfrequencies, or a given variation, an arbitrary number of FRF sets can be generated. 

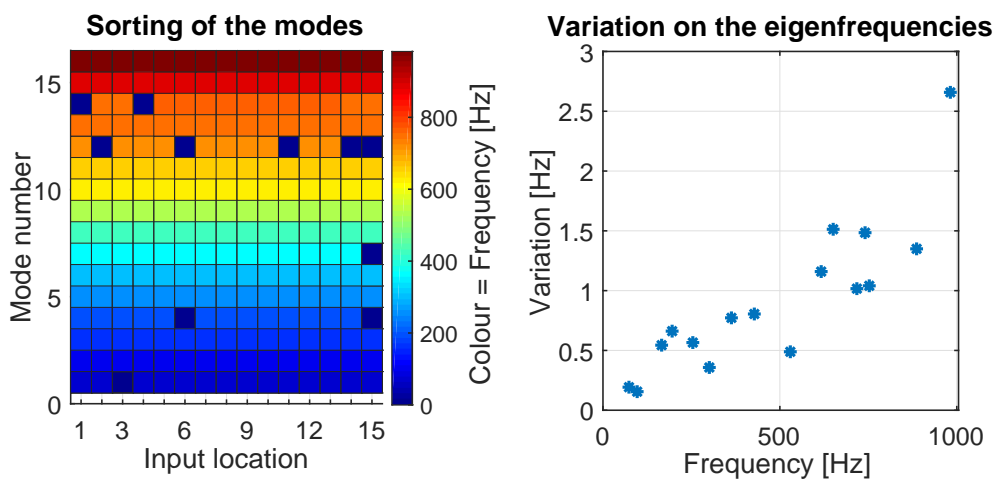

Figure 19: Hammer measurement: Sorting and variation of the eigenfrequencies

Experiments: FRF from 21001 to 31002: new vs. standard approach
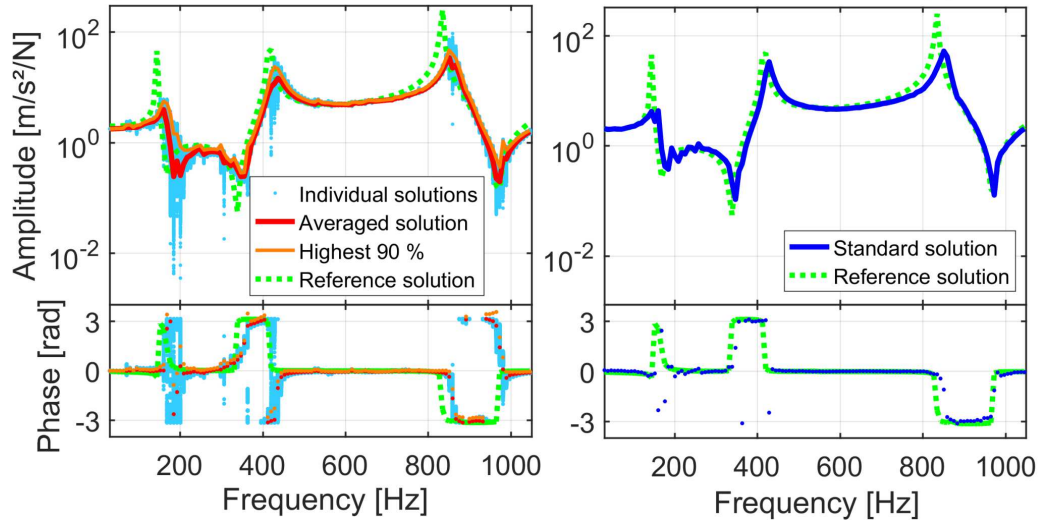

Figure 20: Hammer excitation: new approach (left, 7/9 sing. vals) vs. standard approach (right, $7 / 9$ sing. vals) (1)

Since it is not known which of the different measurement runs provides the measurement that is closest to reality, a large number of calculations is performed, of which the average or upper $90 \%$ curve provides a good estimation of the decoupled FRF.

It has been shown that the sorting of the eigenfrequencies is robust as it doesn't require all modes to be consistently identified to obtain good results. 
Experiments: FRF from 21001 to 31007 : new vs. standard approach
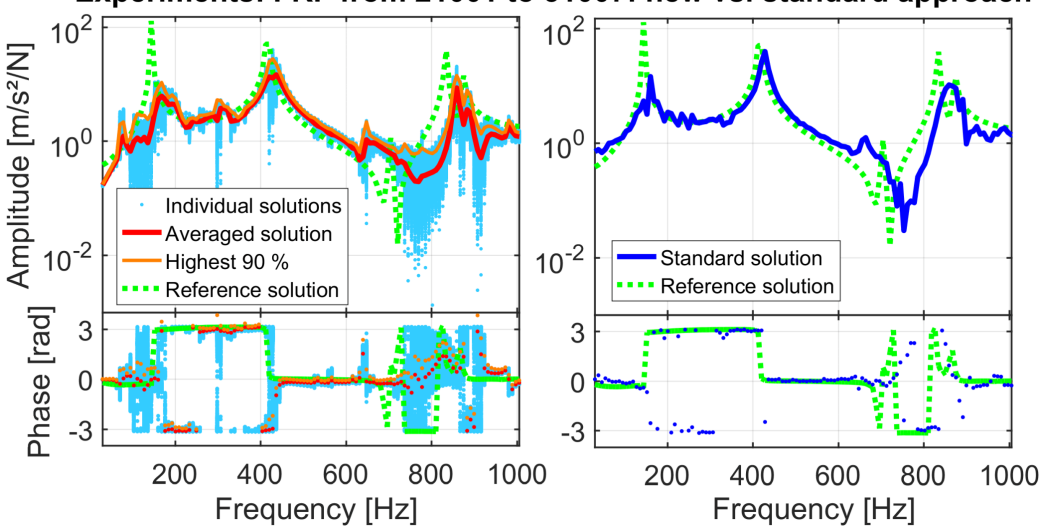

Figure 21: Hammer excitation: new approach (left, 7/9 sing. vals) vs. standard approach (right, $7 / 9$ sing. vals) (2)

However, the approximation of the original FRF is vital and remains difficult to control, especially if eigenfrequencies are present just outside the investigated frequency range. This can cause modelling errors to be more detrimental than measurement errors.

The new method is tested on both numerical models as well as experimental data and compared to the more often used method that makes use of raw FRFs. First the influence of different types of noise is investigated for both methods on an analytical model. Secondly, the robustness of the new method to noise and measurement errors is demonstrated on numerical FRFs of an FE-model of a test rig.

Finally, the method is tested on the physical test rig. In the first measurement, the FRF matrices are measured with a roving shaker, introducing considerable inconsistencies in the data. In this case, the new method improved the results. In the second measurement, FRFs were obtained using a roving hammer, resulting in very high quality FRFs. Here, the new method provided similar, good results as the standard approach for most FRFs proving that modelling errors can be small enough not to influence the decoupling results too much. 


\subsection{Future work}

FRF-based substructure decoupling remains very sensitive to measurement and/ or modelling errors. A modal model can filter noise from the FRF matrices but the fitting of the model on the FRFs remain difficult to control. Other modal parameter estimation methods, like e.g. the MLMM estimation method presented by El-Kafafy et al. [33] may improve the approximation as the modal parameters are estimated iteratively, allowing a better approximation to the original FRF.

In this paper, the distribution of the different solutions of the decoupled FRFs is assumed to be normal. As an alternative to the average or upper $90 \%$ curve, it can be investigated what type of distribution fits best on the decoupled solutions to allow a more reliable estimate of the most likely solution.

During the simulations as well as the measurements, it was observed that FRFs that are low in amplitude, especially those between lowly coupled directions, are most sensitive to modelling and measurement errors. A criterion could be established to determine which FRFs are the most likely to be successfully decoupled.

More research could focus on establishing a practical criterion for the optimal selection of measurement locations that ensures the lowest condition number possible.

\section{Acknowledgements}

The Research Fund KU Leuven is gratefully acknowledged for its support. This work benefits from the Belgian Programme on Inter university Attraction Poles, initiated by the Belgian Federal Science Policy Office (DYSCO).

The Flanders Innovation \& Entrepreneurship Agency is gratefully acknowledged for its support within the MBSE project as well as the research of T. Tamarozzi. 


\section{References}

[1] D. de Klerk, D. J. Rixen, S. Voormeeren, General framework for dynamic substructuring: history, review and classification of techniques, AIAA journal 46 (5) (2008) 1169-1181.

[2] W. D'Ambrogio, A. Fregolent, Direct decoupling of substructures using primal and dual formulation, in: Linking Models and Experiments, Volume 2, Springer, 2011, pp. 47-76.

[3] P. Ind, D. Ewins, Impedance based decoupling and its application to indirect modal testing and component measurement: a numerical investigation, Proceedings of 21st IMAC (2003) 9.

[4] W. D'Ambrogio, A. Fregolent, Decoupling of a substructure from modal data of the complete structure, in: Proceedings of ISMA, Vol. 2004, 2004, pp. 2693-2706.

[5] M. S. Allen, R. L. Mayes, E. J. Bergman, Experimental modal substructuring to couple and uncouple substructures with flexible fixtures and multipoint connections, Journal of Sound and Vibration 329 (23) (2010) 48914906.

[6] R. L. Mayes, M. S. Allen, D. C. Kammer, Eliminating indefinite mass matrices with the transmission simulator method of substructuring, in: Topics in Experimental Dynamics Substructuring and Wind Turbine Dynamics, Volume 2, Springer, 2012, pp. 21-31.

[7] D. C. Kammer, M. S. Allen, R. L. Mayes, Formulation of an experimental substructure model using a craig-bampton based transmission simulator, Journal of Sound and Vibration 359 (2015) 179-194.

[8] W. D'Ambrogio, A. Fregolent, Prediction of substructure properties using decoupling procedures, in: Proceedings of EURODYN, 2005. 
[9] W. D'Ambrogio, A. Sestieri, A unified approach to substructuring and structural modification problems, Shock and Vibration 11 (3-4) (2004) 295309.

[10] P. Sjövall, T. Abrahamsson, Substructure system identification from coupled system test data, Mechanical Systems and Signal Processing 22 (1) (2008) 15-33.

[11] W. D'Ambrogio, A. Fregolent, Decoupling procedures in the general framework of frequency based substructuring, in: Proceedings of 27th IMAC. Orlando (USA), 2009.

[12] S. Voormeeren, D. Rixen, A dual approach to substructure decoupling techniques, in: Structural Dynamics, Volume 3, Springer, 2011, pp. 601-616.

[13] S. Voormeeren, D. Rixen, A family of substructure decoupling techniques based on a dual assembly approach, Mechanical Systems and Signal Processing 27 (2012) 379-396.

[14] W. D'Ambrogio, A. Fregolent, Are rotational dofs essential in substructure decoupling?, in: Proceedings of the 32nd International Modal Analysis Conference (IMAC), Springer, 2014, pp. 27-36.

[15] P. Peeters, T. Tamarozzi, F. Vanhollebeke, W. Desmet, A robust approach for substructure decoupling, in: Proceedings of the International Conference on Noise and Vibration Engineering ISMA, 2014, pp. 3907-3921.

[16] W. D'Ambrogio, A. Fregolent, Ignoring rotational dofs in decoupling structures connected through flexotorsional joints, in: Dynamics of Coupled Structures, Volume 4, Springer, 2015, pp. 57-69.

[17] A. Fregolent, et al., Substructure decoupling without using rotational dofs: Fact or fiction?, Mechanical Systems and Signal Processing 72 (2016) 499512. 
[18] K. Sanliturk, O. Cakar, Noise elimination from measured frequency response functions, Mechanical Systems and Signal Processing 19 (2005) 615631. doi:10.1016/j.ymssp.2004.04.005.

[19] B. Peeters, H. Van der Auweraer, P. Guillaume, J. Leuridan, The polymax frequency-domain method: a new standard for modal parameter estimation?, Shock and Vibration 11 (3-4) (2004) 395-409.

[20] P. Guillaume, P. Verboven, S. Vanlanduit, H. Van Der Auweraer, B. Peeters, A poly-reference implementation of the least-squares complex frequency-domain estimator, in: Proceedings of IMAC, Vol. 21, 2003, pp. 183-192.

[21] P. Peeters, S. Manzato, T. Tamarozzi, W. Desmet, Substructure decoupling with noisy and inconsistent data, in: Proceedings of the International Conference on Noise and Vibration Engineering ISMA 2016, 2016, pp. 3907-3921.

[22] P. van der Valk, J. van Wuijckhuijse, D. de Klerk, A benchmark test structure for experimental dynamic substructuring, Structural Dynamics, Volume 3: Proceedings of the 28th IMAC, A Conference on Structural Dynamics, 2010 (2011) 1113-1122.

[23] P. Avitabile, M. Peres, Excitation techniques - dos and don'ts, in: IMACXXVI, A Conference \& Exposition on Structural Dynamics, 2012.

URL http://www.modalshop.com/filelibrary/Excitation Techniques_IMAC_30_by_Pete_Avitable.pdf

[24] D. J. Rixen, How measurement inaccuracies induce spurious peaks in frequency based substructuring, in: IMAC-XXVI, A Conference \& Exposition on Structural Dynamics, 2008.

[25] W. DAmbrogio, A. Fregolent, Optimal replacement of coupling dofs in substructure decoupling, in: Dynamics of Coupled Structures, Volume 4, Springer, 2016, pp. 77-90. 
[26] H. Van Der Auweraer, P. Guillaume, P. Verboven, S. Vanlanduit, Application of a fast-stabilizing frequency domain parameter estimation method, Journal of dynamic systems, measurement, and control 123 (4) (2001) 651658.

[27] J. Lanslots, B. Rodiers, B. Peeters, Automated pole-selection: proof-ofconcept and validation, in: Proceedings, international conference on noise and vibration engineering (ISMA), Katholieke Universiteit Leuven, Belgium, 2004.

[28] B. Peeters, J. Lau, J. Lanslot, H. v. d. Auweraer, Automatic modal analysismyth or reality? Proceedings of the International Modal Analysis Conference (IMAC), 42-3 (2008), pp. 17-21.

[29] W. Heylen, S. Lammens, P. Sas, Modal Analysis Theory and Testing, Katholieke Universiteit Leuven Departement Werktuigkunde, 1995.

[30] R. Allemang, D. Brown, A complete review of the complex mode indicator function CMIF with applications, in: Proceedings, international conference on noise and vibration engineering (ISMA), Katholieke Universiteit Leuven, Belgium, Vol. 38, 2006, pp. 36-44.

[31] R. J. Allemang, The modal assurance criterion-twenty years of use and abuse, Sound and vibration 37 (8) (2003) 14-23.

[32] W. Heylen, P. Avitabile, Correlation considerations. part 5.(degree of freedom correlation techniques), in: SPIE proceedings series, Society of PhotoOptical Instrumentation Engineers, 1998, pp. 207-214.

[33] M. El-Kafafy, B. Peeters, P. Guillaume, Incorporating real modes and reciprocity constraints in the iterative MLMM modal parameter estimation method, in: Proceedings, international conference on noise and vibration engineering (ISMA), Katholieke Universiteit Leuven, Belgium, 2016, pp. 2933-2946. 